\title{
THE MAGNETIC PROPERTIES OF TEXTURED FINE PARTICLE SYSTEMS
}

\author{
R. W. CHANTRELL \\ School of Physics and Astronomy, Lancashire Polytechnic, Preston, Lancashire, \\ PR1 2TQ, U.K.
}

The magnetic properties of fine particle systems are strongly dependent on two types of texture. The first is an orientational texture characteristic of the angular dispersion of the easy anisotropy axes and the second is a spatial texture characteristic of the physical microstructure of the system. The effects of texture on the properties of both fluid and solid dispersions of fine particles can be very pronounced. The paper reviews some recent studies illustrating these effects, with particular emphasis on the behaviour of recording media and magnetic fluids.

KEY WORDS Fine particles, solid dispersions, liquid dispersions, orientational texture, spatial texture, recording media, magnetic fluids.

\section{INTRODUCTION}

Dispersions of fine magnetic particles are probably most widely recognised in the form of particulate recording media, which are of enormous technical importance. At present there is considerable interest in the behaviour of these systems since there exist many fundamental scientific problems to be solved if particulate media are to reach their full potential. Another important class of materials are colloidal dispersions of ferromagnetic particles often referred to as ferrofluids.

Both types of material are comprised of single domain particles, which differ dramatically from bulk materials in their magnetic behaviour. The properties of bulk materials are dominated by the behaviour of magnetic domains. These are formed as a result of the competing requirements of the exchange energy which favours parallel alignment of the atomic magnetic moments and the magnetostatic energy which is minimised in a completely demagnetised sample. Domains are regions of the material which are spontaneously magnetised but which can orient at random on a macroscopic scale in order to reduce the magnetostatic energy. The magnetostatic energy is proportional to the sample volume. As the sample size is reduced the magnetostatic energy decreases until a critical volume is reached at which it is no longer energetically favourable to form magnetic domains. Particles smaller than this size consist of a single domain only.

The magnetic properties of bulk materials are dominated primarily by the fact that domain wall motion is relatively easy. In single domain particles this is not a possibility. Generally they possess a large magnetic anisotropy, which can be crystalline in origin or result from the anisometric shape of the particles. Magnetisation reversal must take place against an energy barrier determined by the magnetic anisotropy. As first shown by Stoner and Wohlfarth ${ }^{1}$ this results in a 
large coercive force of the order of the anisotropy field $H_{k}=2 K / I_{s b}$ where $K$ is the anisotropy constant and $I_{s b}$ is the saturation magnetisation of the bulk material. In practice the coercivity of fine particle materials is generally smaller than $H_{k}$ for the following reasons:

- The effects of thermal agitation. At a finite temperature there is a possibility of thermally activated transitions over the energy barrier. As the particle size decreases this becomes more important. The coercivity vanishes at a volume $V_{p}$. Below this the particles exhibit thermal equilibrium (superparamagnetic) behaviour.

- Incoherent reversal modes, such as curling, give a lower magnetostatic energy than the Stoner-Wohlfarth coherent rotation mechanism and tend to lower the coercive force.

There are two further fundamental reasons for the reduction in coercivity which are central to this paper.

- The above value of the coercivity is valid only for a system whose easy axes are aligned parallel to the applied field. A distribution of easy axes results in a reduction of the coercivity as shown in Ref. 1.

- Magnetostatic interactions between the particles tend to reduce the coercivity in general

The first factor can be described as an orientational texture representing the distribution function of the easy axis orientations. The second is sensitive to the magnetic microstructure of the system which may be thought of as a spatial texture. In this paper we shall consider the effcts of both types of texture on the magnetic properties of fine particle systems, specifically weakly interacting systems of small cobalt particles and more strongly interacting systems in the form of recording media where the spatial texture depends upon the detailed preparation conditions (principally the degree of dispersion and the external applied field). These are of course solid dispersions. The effects of texture on the fluid dispersions (ferrofluids) is rather more subtle but none the less interesting, and will also be considered in detail.

\section{COLLOIDAL DISPERSIONS}

It is useful to consider the properties of colloidal dispersions in the first instance since the texture can often be frozen into solid systems. Thus the present section also forms an introduction to the ideas of texture in solids. Orientational texture in ferrofluids is produced by the particle anisotropy. This has no bearing on the magnetic properties of the dispersion since the rotational freedom of the particles effectively decouples the anisotropy from the magnetisation process. ${ }^{2,3}$ However, the anisotropy energy gives rise to a coupling between the magnetic moment and easy axes of the particle which tends to rotate the easy axis into the field direction. The competing effect of thermal agitation results in a distribution of the easy axes or orientational texture with the field direction as a symmetry axis.

In the fluid state this texture can be observed in the form of anisotropic optical properties, for example a field induced birefringence, and in the mag- 
netodielectric effect, which is an anisotropy in the dielectric properties of the fluid. Often these effects are related to the orientational texture via the function $\left\langle\cos ^{2} \theta\right\rangle$ which has previously been calculated for ferrofluids. ${ }^{4,5}$

The calculation assumes the system to be in thermal equilibrium and uses Boltzmann statistics to determine the orientational distribution. For convenience we use a coordinate system centred on the magnetic moment of a particle as shown in Figure 1. The energy of the particle consists of two terms; the anisotropy energy and the energy of interaction with the magnetic field. In the chosen coordinate system the energy is:

$$
E_{p}=K V \sin ^{2} \omega-\mu H \cos \phi,
$$

where the angles $\omega$ and $\phi$ are defined in Figure 1 and $\mu$ is the magnetic moment of the particle. The magnetic field has an aligning influence on the magnetic moment. The coupling between the easy axis and magnetic moment results in a distribution of $\theta$ about the field direction which determines an average value of $\cos ^{2} \theta$ given by

$$
\left\langle\cos ^{2} \theta\right\rangle=\frac{\int_{0}^{2 \pi} \int_{0}^{2 \pi} \int_{0}^{\pi} \sin \omega \sin \phi \cos ^{2} \theta \exp \left(-E_{p} / k T\right) d \omega d \phi d \xi}{\int_{0}^{2 \pi} \int_{0}^{2 \pi} \int_{0}^{\pi} \sin \omega \sin \phi \exp \left(-E_{p} / k T\right) d \omega d \phi d \xi}
$$

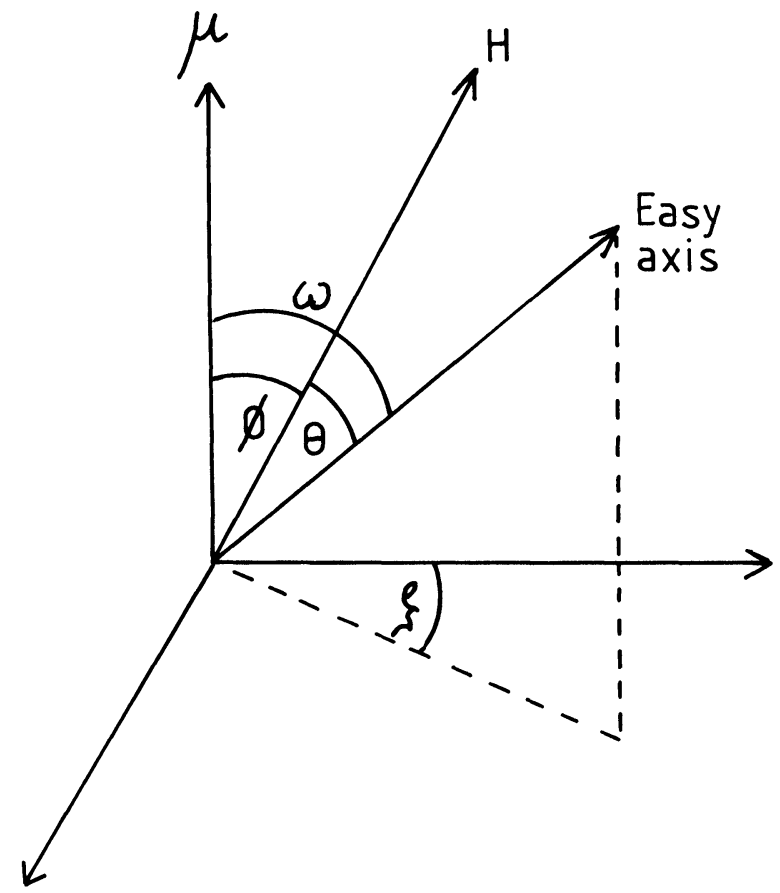

Figure 1 Coordinate system used in the calculation. 
Evaluation of the integrals is carried out (4), giving the result

$$
\left\langle\cos ^{2} \theta\right\rangle=\left(1-\frac{2 L(b)}{b}\right) \Theta+\frac{L(b)}{b}(1-\Theta) .
$$

The function $\Theta$ represents the strength of the coupling between the easy axes and magnetic moment, and depends on the ratio of the anisotropy energy to the thermal energy $k T$ via the relation:

where $\alpha=K V / k T$ and

$$
\Theta=\frac{\exp (\alpha)}{\alpha I(\alpha)}-\frac{1}{2 \alpha}
$$

$$
I(\alpha)=\int_{-1}^{+1} \exp \left(\alpha x^{2}\right) d x
$$

A useful approximation to $\Theta$, valid in the limit $\alpha \rightarrow \infty$ is

$$
\Theta=1-1 / \alpha \text {. }
$$

The coupling function is shown in Figure 2.

The function $\Theta$ essentially determines the anisotropic behaviour of ferrofluids in the absence of interparticle interactions. The birefringence of ferrofluids is a very good example which has been extensively investigated. ${ }^{5,6}$ A closely related measurement is the magnetodielectric effect ${ }^{2,7,8}$ which is a field-induced anisotropy in the relative permittivity of the ferrofluid. This is used as an example here because there also exist calculations which relate this effect to the spatial texture induced by interparticle interactions, as will be described later in Section 3.

It can be shown ${ }^{2}$ that the relative permittivity measured parallel and perpendicular to an applied field $\varepsilon_{r}^{\prime}$ and $\varepsilon_{r}^{\prime \prime}$ are given by

$$
\begin{gathered}
\varepsilon_{r}^{\prime}=1+f \gamma+f \delta \int_{0}^{\infty}\left\langle\cos ^{2} \theta\right\rangle F(V) d V, \\
\varepsilon_{r}^{\prime \prime}=1+f \gamma+f \delta\left[1-\int_{0}^{\infty}\left\langle\cos ^{2} \theta\right\rangle F(V) d V\right] .
\end{gathered}
$$

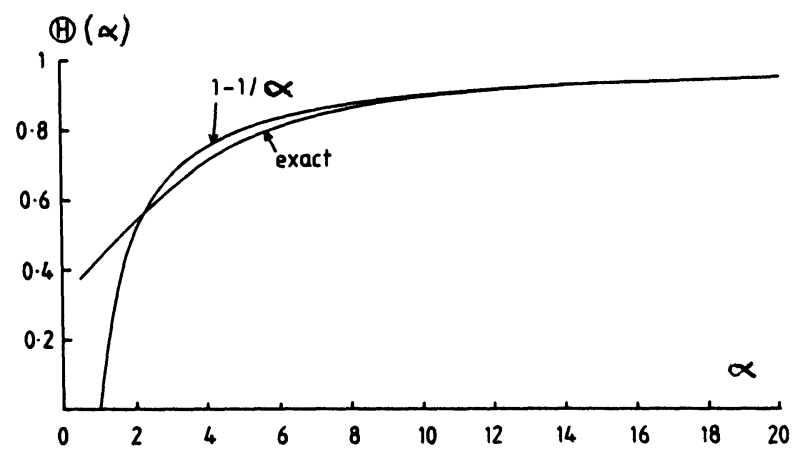

Figure 2 The variation of the coupling function with the parameter $\alpha=K V / k T$. 


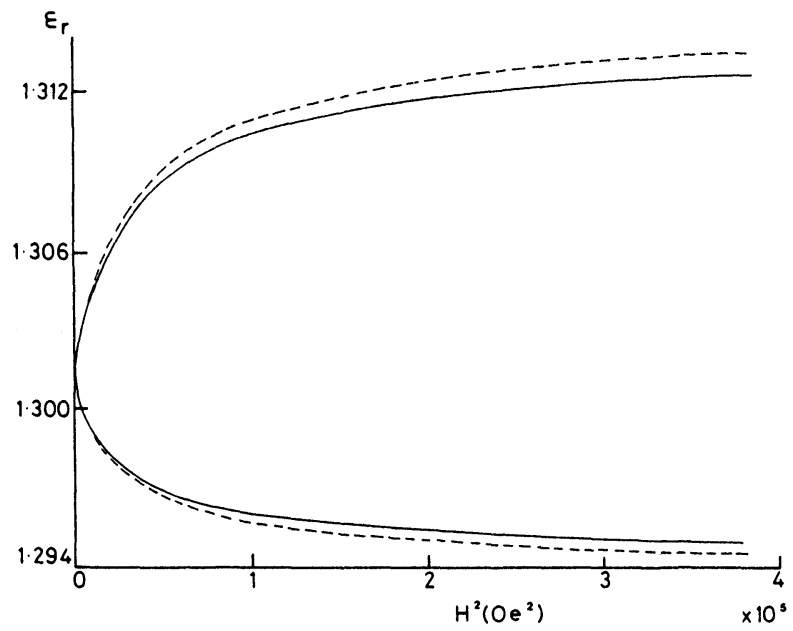

Figure 3 The variation of $\varepsilon_{r}$ with (applied field) for cobalt particles having a median volume of $2 \times 10^{-19} \mathrm{~cm}^{3}$. Full line-calculation given here. Broken line-perfect coupling approximation.

Here, $f$ is the volumetric packing fraction of the particles and $\gamma$ and $\delta$ are constants dependent on the relative permittivities of the particles and base fluid and the shape of the particles. ${ }^{2} F(V)$ is a distribution function taking into account the spread of particle volumes which is an important factor in all ferrofluids.

Typical results for a dispersion of cobalt particles are shown in Figure 3. A lognormal size distribution with median volume $2 \times 10^{-19} \mathrm{~cm}^{3}$ and standard deviation $\sigma=0.3$ was assumed. The lognormal size distribution has the form

$$
F(y)=\frac{1}{\sqrt{2 \pi} \sigma y} \exp \left(-(\operatorname{Ln}(y))^{2} / 2 \sigma^{2}\right),
$$

where $y=D / D_{V}$ with $D_{V}$ the median diameter of the distribution. The particles have a value of $K V / k T=5$. Also shown in Figure 3 is the behaviour which would be expected for infinite coupling. As might be expected this gives a larger anisotropy, indicating that for real systems the full theory outlined here is important in the investigation of textured systems.

It is possible in principle to freeze in the induced texture, either by cooling through the melting point of the base fluid or by using the polymerisation technique of Liebert et al. ${ }^{9}$ The properties of textured systems produced in this manner are of some interest and will be discussed in Section 4. Before moving on to examples of this type, however, we consider the effects of dipolar magnetostatic interactions on the behaviour of colloidal dispersions.

\section{SPATIAL TEXTURE IN COLLOIDAL DISPERSIONS}

Because of the requirement of colloidal stability it is necessary to minimise the particle size used in ferrofluids. However, because of the long-ranged nature of the dipolar interaction a colloidal magnetic dispersion can never be completely 
free of its effects. An important effect of the dipolar interaction is the production of a degree of spatial ordering of the particles which can be considered as a "Spatial Texture." This can be anisotropic in the presence of an applied magnetic field. Here we give the results of a Monte-Carlo model of particle interactions and apply these to the problem of the magnetodielectric effect.

The Monte-Carlo method essentially entails the generation of a Markov chain of points in phase space where the transition probability between neighbouring points is taken to be $\exp (-\Delta E / k T)$ where $\Delta E$ is the energy difference between the points. In practice a representative cell of $N(\sim 1000)$ particles is set up, and each particle given a random displacement in its coordinates (including its associated magnetic moment). The energy difference $(\Delta E)$ between the two positions is calculated and the trial move accepted with a probability $\exp (-\Delta E / k T)$. Periodic boundary conditions are used to extend the cell, i.e. if a particle leaves the cell it is assumed to re-enter on the opposite side. After many such Monte-Carlo moves/particle the system can be shown ${ }^{10}$ to evolve into a thermal equilibrium state, characterised by a Boltzmann energy distribution.

For the case of strongly interacting particles the Monte-Carlo approach has been shown ${ }^{11}$ to result in a spatial texture whose form and extent is dependent upon the applied field. The spatial texture can be characterised by calculating the pair distribution function $g(\vec{r})$ defined as the average number density at a distance $\vec{r}$ from a given particle, normalised relative to the average number density in the system. Typical results for a two dimensional simulation are given in Figure 4 for cobalt particles of $15 \mathrm{~nm}$ diameter in applied fields of zero and $10 \mathrm{kOe}$. There is extensive order apparent in both configurations. In zero field closed loop structures are produced which break up into highly anisotropic chain-like configurations as shown in (b). Figure 5 shows the corresponding $g(\vec{r}) \cdot g(\vec{r})$ is
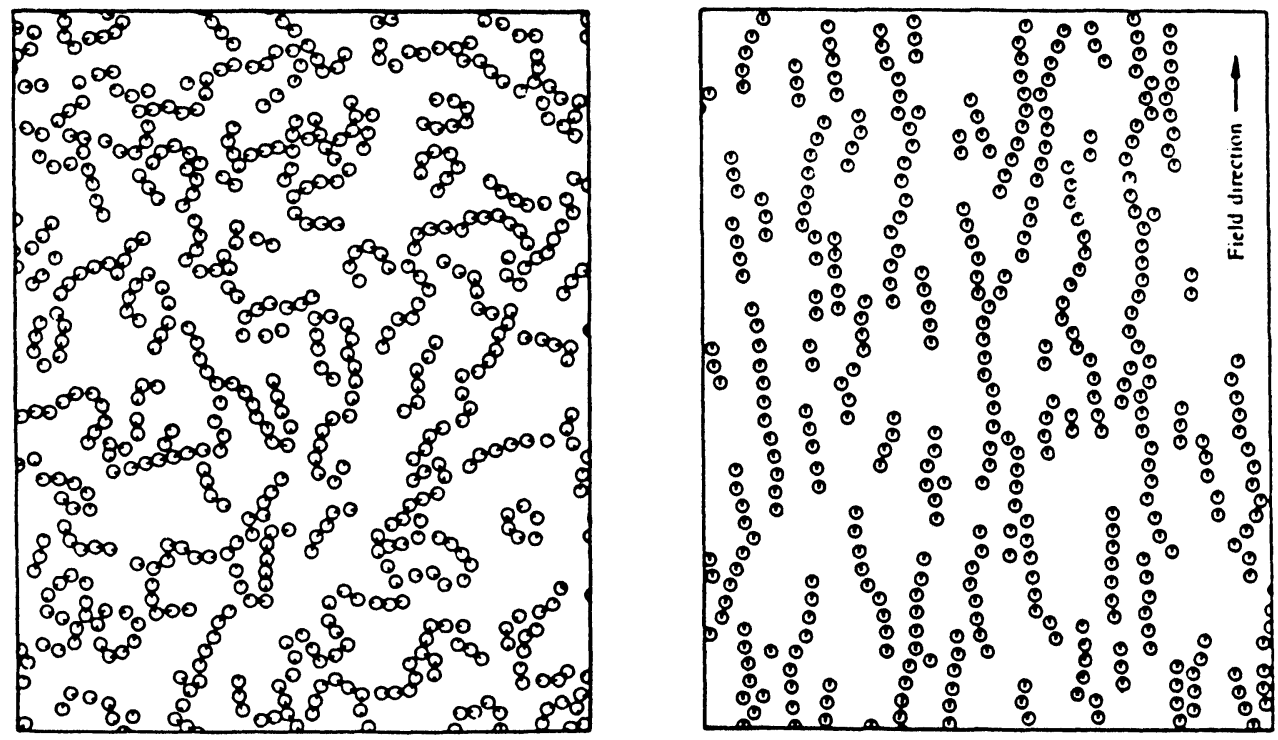

Figure 4 Configurations for $15 \mathrm{~nm}$ cobalt particles in zero field (a) and in a field of $10 \mathrm{kOe}(\mathrm{b})$. 


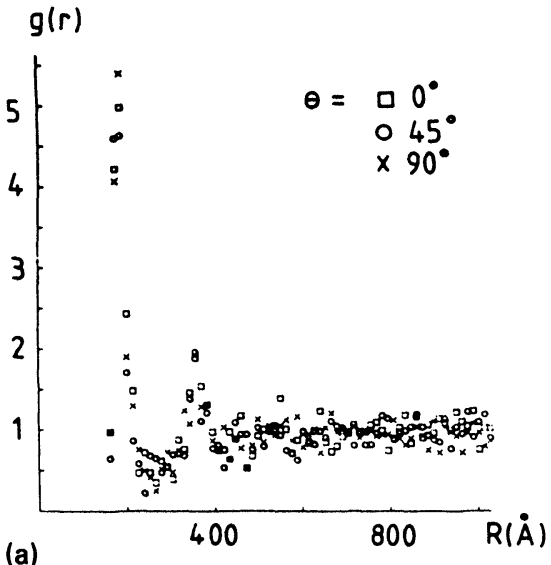

(a)

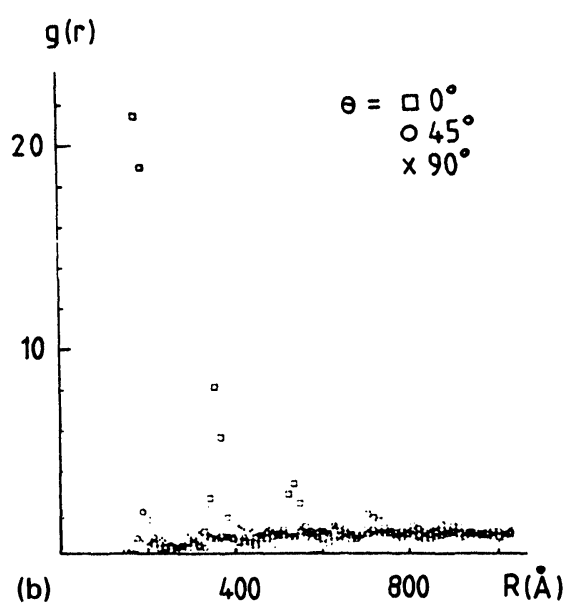

Figure $5 g(r, \theta)$ for $15 \mathrm{~nm}$ cobalt particles for zero field (a) and a field of $10 \mathrm{kOe}(\mathrm{b})$. The anisotropy in the configuration is apparent.

given as $g(r, \theta)$ where $\theta$ is the angle the line joining the particle centres makes with the applied field. The anisotropy in the configuration in a field of $10 \mathrm{kOe}$ is apparent in Figure 5, where $g(r, \theta)$ has a large nearest neighbour peak at $\theta=0$, with very little correlation apparent at $\theta=\pi / 2$.

It seems reasonable to expect that the spatial texture could contribute to the anisotropic properties of ferrofluids. We shall illustrate this with reference to the magnetodielectric effect.

Consider two particles oriented at some angle $\theta$ to the measurement direction and separated by a distance $r$. In this situation it has been shown ${ }^{12,13}$ that electric interactions between particles give rise to an enhancement of the relative permittivity by an amount:

$$
\Delta \varepsilon_{r}=\frac{\left(\varepsilon_{2}-\varepsilon_{1}\right)^{2} f V\left(\frac{3}{2} \cos ^{2} \theta-\frac{1}{2}\right)}{\left.1+\left(\varepsilon_{2}-\varepsilon_{1}\right) / 3 \varepsilon_{1}\right)^{2} 2 \pi r^{3}},
$$

where $\varepsilon_{1}$ and $\varepsilon_{2}$ are the relative permittivities of the fluid and particles respectively, $f$ is the volumetric packing fraction and $V$ the particle volume. In practice there will be a distribution of $\theta$, and $\Delta \varepsilon_{r}$ will be determined by the order parameter $S=\left\langle\frac{3}{2} \cos ^{2} \theta-\frac{1}{2}\right\rangle$, where \langle\rangle denotes an ensemble average. $S$ can be calculated using the pair distribution function which leads to:

$$
\Delta \varepsilon_{r}=\frac{\left(\varepsilon_{2}-\varepsilon_{1}\right)^{2} f V}{\left.1+\left(\varepsilon_{2}-\varepsilon_{1}\right) / 3 \varepsilon_{1}\right)^{2}} \int g(\vec{r})\left(\frac{3}{2} \cos ^{2} \theta-\frac{1}{2}\right) \frac{d \vec{r}}{r} .
$$

The 2-D simulations mentioned previously have been used by Ayoub et al. ${ }^{13}$ to calculate the magnetodielectric anisotropy for $8 \mathrm{~nm}$ diameter cobalt particles. In $2-D$ the angular part of the equivalent order parameter is $S=\cos ^{2} \theta-1 / 2$. Data for this simulation are given in Figure 6 which shows the variation of $\Delta \varepsilon_{r}$ with applied field. It can be seen that particle interactions give rise to a significant dielectric anisotropy, which has a form similar to that predicted for orientational texture. A further study $^{14}$ using an integral equation approach has also shown 


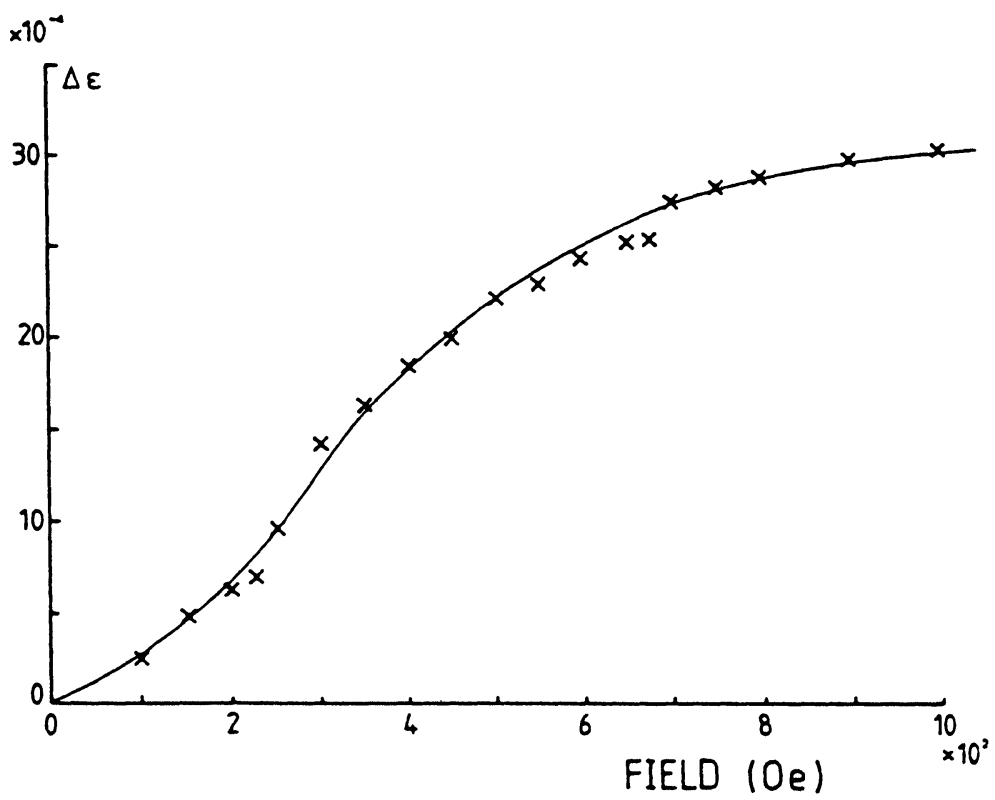

Figure 6 The variation of $\Delta \varepsilon_{r}$ with field for an interacting ferrofluid.

that anisotropic spatial order can account for the magnetodielectric effect and presumably for other anisotropic properties of ferrofluids. In fact, in highly stable systems it is likely that both effects are present and contribute to the experimental values. The anisotropic properties of ferrofluids are of some interest and considerable research is still being carried out in this area.

\section{DISPERSIONS OF ULTRAFINE PARTICLES}

\section{Superparamagnetic Behaviour}

In this section we discuss the effects of texture on the behaviour of superparamagnetic particles in the solid state. We begin by considering the behaviour of a particle of volume $V$ with uniaxial anisotropy and a first order anisotropy constant $K$. In the solid state the magnetic moment of the particle must rotate with respect to the particle and hence the anisotropy energy makes an important contribution to the magnetic behaviour. Here we shall consider those particles whose energy barriers are small enough for thermal agitation to be effective in allowing the magnetic sub-system to attain thermal equilibrium within the timescale of the experiment. This is the case for superparamagnetic particles which have volumes smaller than a critical value given by $K V_{p} / k T=25 .{ }^{15}$ It is convenient to carry out the calculation of the initial susceptibility in coordinate system centred on the particle easy axis, such a coordinate system being given in Figure 7. 


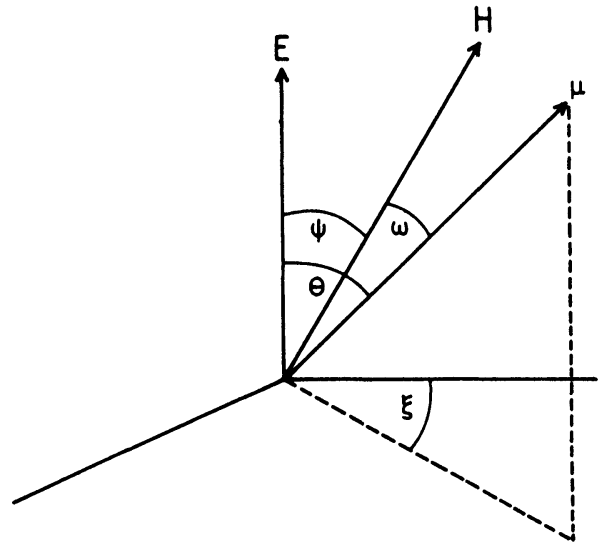

Figure 7 Coordinate system used in the calculation of the susceptibility of a single particle. Note that $E$ denotes the easy axis direction.

In this coordinate system the energy of a particle can be written

$$
E=K V \sin ^{2} \theta-\mu H \cos \omega
$$

with the angle $\omega$ determined by the relation

$$
\cos \omega=\cos \theta \cos \psi+\sin \theta \sin \psi \cos \xi .
$$

The partition function of the system is given by

$$
Z=(4 \pi)^{-1} \int_{0}^{2 \pi} \int_{0}^{2 \pi} \sin \theta \exp \left(-\alpha \sin ^{2} \theta+\beta \cos \omega\right) d \theta d \xi,
$$

where $\beta=\mu H / k T$. The initial susceptibility of a system is characteristic of its low field behaviour, where $\beta \ll 1$. In this limit we can approximate $Z$ by expanding the exponential in powers of $\beta$ and retaining only the low order terms. The term in $\beta$ vanishes, so that, correct to order $\beta^{2}$ we have that

where

$$
Z=(4 \pi)^{-1}\left\{2 \pi I_{0}+\frac{1}{2} \pi \beta^{2}\left[I_{0}-\cos ^{2} \psi I_{0}-I_{2}\left(1-3 \cos ^{2} \psi\right)\right]\right\},
$$

$$
I_{n}=\int_{0}^{\pi} \cos ^{n} \theta \sin \theta \exp \left(-\alpha \sin ^{2} \theta\right) d \theta .
$$

The reduced magnetisation of the system (relative to saturation) is given by $I=\partial(\ln Z) / \partial \beta$. Therefore using Eq. (12) we can calculate the reduced susceptibility $\chi_{r}=d I / d H$ which is given by

$$
\chi_{r}=\frac{\mu}{k T}\left(\cos ^{2} \psi+\frac{1}{2}\left\{1-\frac{1}{2 a}-\frac{\exp (\alpha)}{\alpha I(\alpha)}\right\}\right),
$$

where $I(\alpha)$ is given by Eq. (5). Using the definition of the coupling function $\Theta$ (Eq. (4)), the susceptibility of a system can be obtained by averaging over the texture function to give

$$
\chi_{r}=\frac{\mu}{k T}\left[\left\langle\cos ^{2} \psi\right\rangle+\frac{1}{2}\left(1-3\left\langle\cos ^{2} \psi\right\rangle\right)\left(1-3 \Theta\left(\alpha_{m}\right)\right)\right] .
$$


Here the brackets \langle\rangle represent an ensemble average. The effects of the texture on the initial susceptibility are governed by the parameter $\alpha_{m}=K V / k T_{m}$ where $T_{m}$ is the temperature at which the measurement is made. In the limit $\alpha_{m} \rightarrow 0$ the susceptibility appropriate to the Langevin function is recovered.

Here we consider the production of different degrees of texture in the system achieved by cooling slowly through the solidification temperature $T_{f}$ in an applied magnetic field. The degree of texture achieved is governed by the value of the coupling constant $\left(\alpha_{f}\right)$ at the temperature $T_{f}$. This determines the value of the texture function $\left\langle\cos ^{2} \psi\right\rangle$, and hence the susceptibility via Eq. (14). We can generalise this equation by the introduction of a particle size distribution, which leads to the result:

$$
\chi_{r}=\frac{I_{s} \pi D_{v}^{3}}{6 k T} \int_{0}^{\infty} y^{3} G(\alpha) f(y) d y,
$$

where $G(\alpha)$ is given by:

$$
G(\alpha)=\left\langle\cos ^{2} \psi\right\rangle+\frac{1}{2}\left(1-3\left\langle\cos ^{2} \psi\right\rangle\right)\left(1-3 \Theta\left(\alpha_{m}\right)\right) .
$$

For a completely random system $\left\langle\cos ^{2} \theta\right\rangle=1 / 3$ in which case $\chi_{r}$ takes on the Langevin value. The effect of texture calculated using Eqn (15) is shown in Figure 8. The susceptibility is given for two temperatures and the texture is achieved by cooling the ferrofluid through the freezing point of toluene $(180 \mathrm{~K})$. The value of $\mathrm{K}$ is $2 \times 10^{6} \mathrm{erg} / \mathrm{cc}$ which is a typical value for fcc cobalt particles with predominant shape anisotropy.

Clearly texture can have a significant effect on the magnetic properties of colloidal dispersions. The calculation given here assumes that all particles are

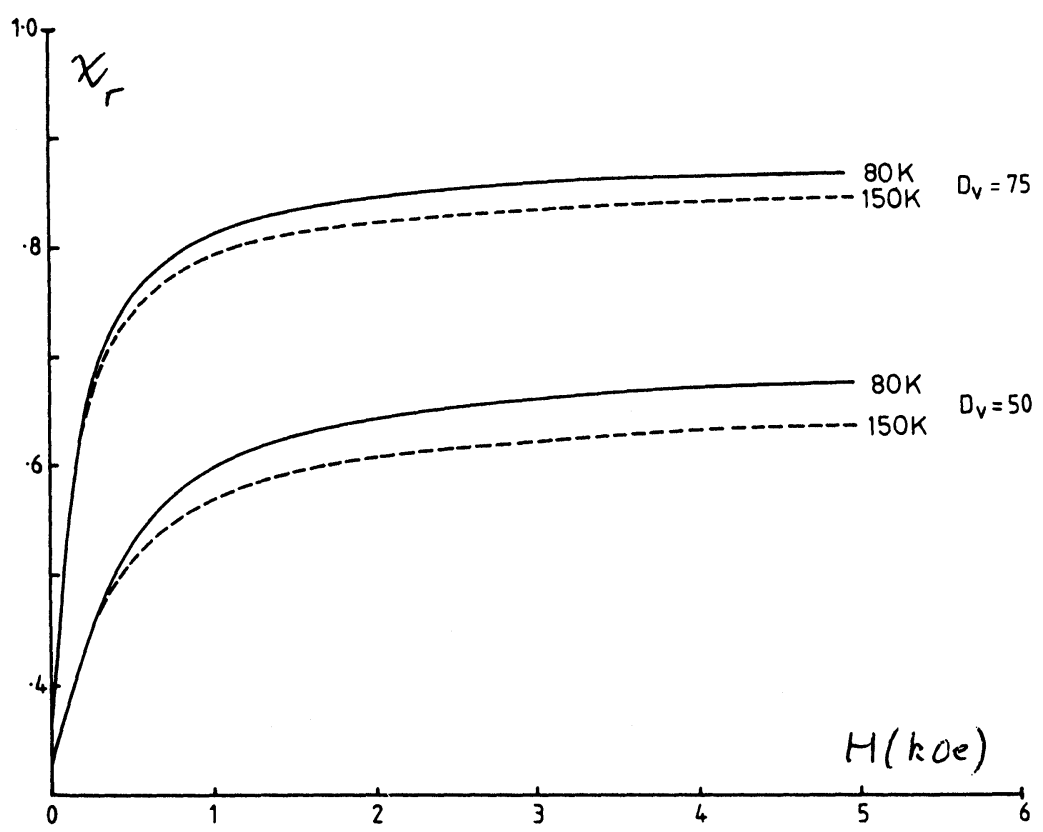

Figure 8 The variation of susceptibility with freezing field for cobalt particles in toluene. 
superparamagnetic, although this is not the case for very large values of $K V / k T$. Under these circumstances the appropriate value for the susceptibility has been given by Ayoub et al. ${ }^{16}$ To a first approximation, however, the susceptibility can be obtained by treating all those particles with diameters greater than the critical diameter for superparamagnetic behaviour $\left(D_{p}\right)$ as making zero contribution. This gives

$$
\chi_{r}=\frac{I_{s} \pi D_{v}^{3}}{6 k T} \int_{0}^{y_{p}} y^{3} G(\alpha) f(y) d y .
$$

where $y_{p}=D_{p} / D_{v}$. In general, if $y_{p}$ is finite the magnetic behaviour begins to exhibit irreversible behaviour and has a measurable coercivity and remanence. These quantities are also strongly dependent upon the texture of the system. This will be investigated further in the following section.

\section{Measurements on Cobalt Particles}

The magnetic behaviour of dispersions of fine cobalt particles is of some interest since the interaction effects can be rather weak. As such they are useful systems for the investigation of the effects of orientational texture. This can be produced as described earlier. Generally these systems consist of mixtures of superparamagnetic particles with those exhibiting non-equilibrium behaviour, this being a result of the particle size distribution. The effects of orientational texture are immediately apparent in the hysteresis loops of the systems as can be seen in Figure 9 (after O'Grady et al. ${ }^{17}$ ). This shows hysteresis loops at $80 \mathrm{~K}$ for $12 \mathrm{~nm}$ diameter cobalt particles. The induced texture is produced by cooling through the freezing point of the base fluid (toluene) in an applied field of $8 \mathrm{kOe}$.

Clearly the effect of field-freezing is to induce an orientational texture which results in an increase in the remanence and coercivity of the material.

The remanence of the system is determined by the irreversible particles, and is given by an integral of the form

$$
\bar{I}_{r}=\int_{y_{p}}^{\infty}\langle\cos \phi\rangle F(y) d y .
$$

This depends on $\langle\cos \phi\rangle$ where $\phi$ is the angle between the easy axis and field direction. This factor is not easy to calculate using the approach given earlier because of the limit on $\phi$ to the range $0<\phi<\pi / 2$. However O'Grady et al. ${ }^{17}$ have given an approximation useful in the limit of strong coupling of $\langle\cos \phi\rangle=$ $L(b)\left(1-\frac{1}{2} a\right)$. This leads to the general expression

$$
\bar{I}_{r}=\int_{0}^{\infty} L(b)(1-1 / 2 a) F(y) d y .
$$

Assuming a lognormal distribution of particle sizes and taking the high field approximation to the Langevin function, Eq. (18) simplifies to

$$
\bar{I}_{r}=2 \bar{I}_{r}(0)-\frac{6 k T_{f} I(\alpha)}{\pi I_{s b} D_{V}^{3}}\left[\frac{1}{H_{0}}+\frac{1}{H_{k}}\right] \text {. }
$$

Here, $H_{0}$ is the field in which the system is frozen and $T_{f}$ is the freezing 

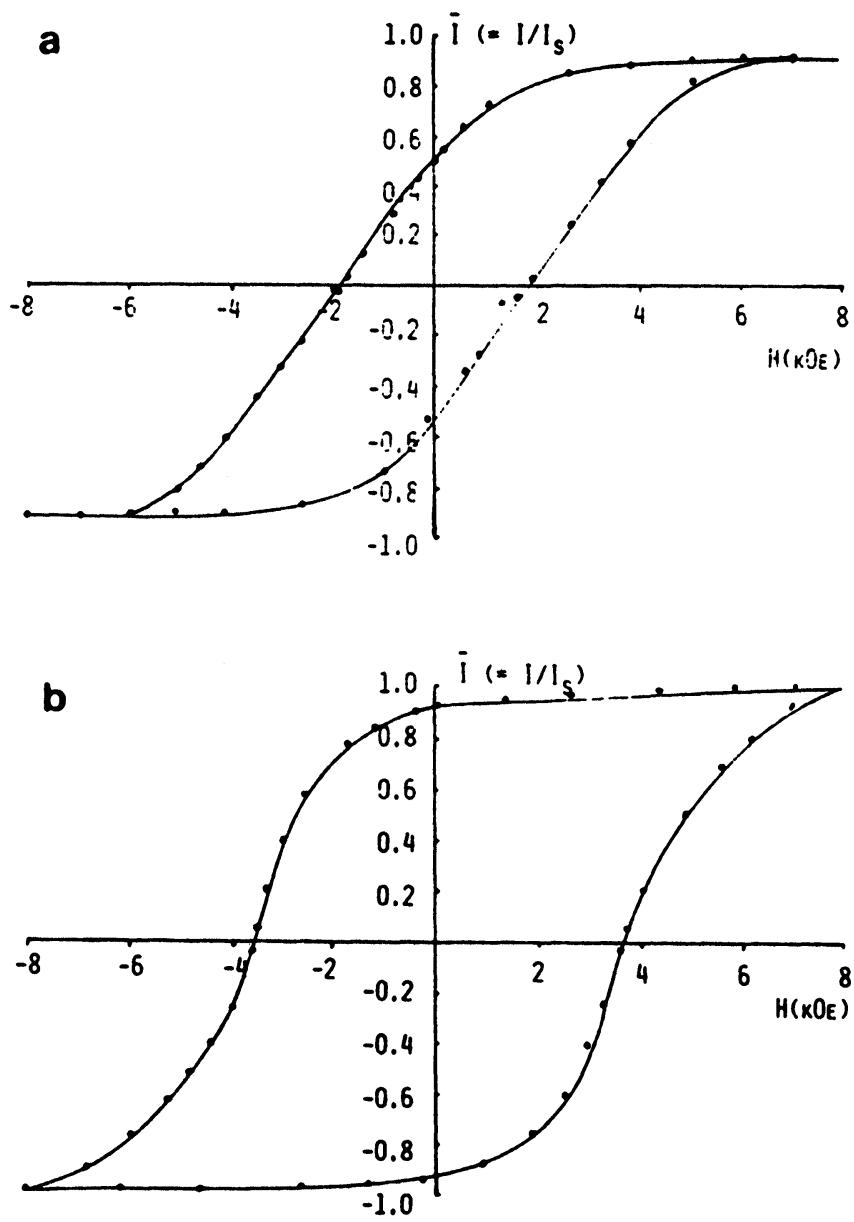

Figure 9 Hysteresis loops for cobalt particles of $12 \mathrm{~nm}$ diameter before (a) and after (b) texturing by freezing in an applied field of $8 \mathrm{kOe}$.

temperature. $I(\alpha)$ is given by Eq. (5). $\bar{I}_{r}(0)$ is the remanence of a system frozen in zero field and consequently randomly oriented. For perfect alignment the remanence is doubled, but in practice thermal agitation reduces this factor. The prediction of Eq. (19) is in agreement with the data of Figure 4.

\section{REMANENCE CURVES OF STRONGLY INTERACTING SYSTEMS}

The behaviour of recording media is strongly influenced by the dipolar interactions between the particles. In this section we present studies of the effects of interactions on the remanence curves. The principal remanence curves are

- The Isothermal Remanent Magnetisation. The reduced remanence (relative to saturation) is denoted $\bar{I}_{r}(H)$ and is obtained by the application of increasingly large fields to an initially demagnetised sample. 
- The DC Demagnetisation Remanence $\bar{I}_{r}(H)$ is obtained by applying increasing negative fields to a sample in the saturated remanence state.

An example of the remanence curves for a powder of $\mathrm{CrO}_{2}$ particles is given in Figure 10 (Spratt et al. ${ }^{18}$ ). Both of these curves can be described in terms of a Switching Field Distribution (SFD) $f(H) d H$ which gives the fraction of particles reversing their magnetisation in fields between $H$ and $H+d H$. The IRM is thus given simply by:

$$
I_{r}(H)=\int_{0}^{H} f(H) d H .
$$

Equation (20) shows that differentiation of the IRM curve gives the SFD directly. The SFD calculated using the IRM data of Figure 10 is shown in Figure 11. In principle the dc demagnetisation remanence can also be calculated using the same SFD. This gives rise to the Wohlfarth ${ }^{19}$ relation between $I_{d}$ and $I_{r}$ :

$$
I_{d}(H)=1-2 I_{r}(H) .
$$

In practice this relation is rarely obeyed experimentally. This was shown to be the case by $\mathrm{Henkel}^{20}$ who investigated plots of $I_{d}$ vs $I_{r}$ for CuNiFe. These were generally found to be non-linear, in disagreement with the predictions of Eq. (21). More recently, Gaunt et al. ${ }^{21}$ have found linear behaviour for PtCo and non-linear behaviour for CuMn. Pinkerton ${ }^{22}$ and Pinkerton and van Wingerden ${ }^{23}$

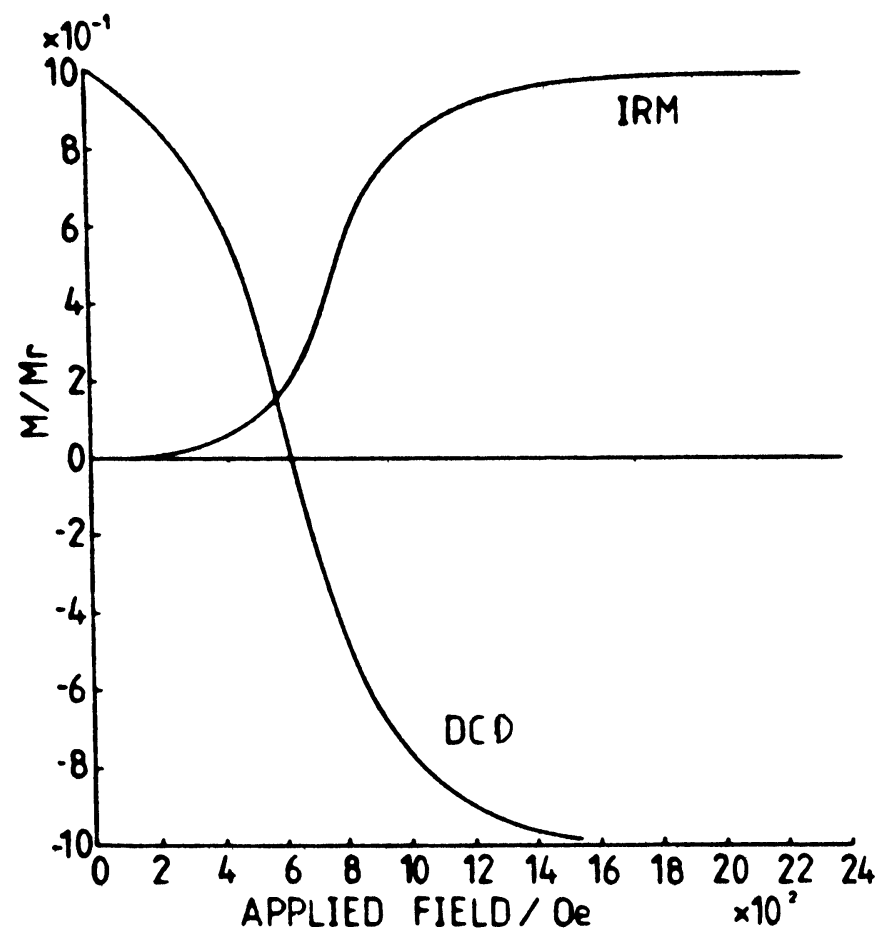

Figure 10 Remanence Curves for $\mathrm{CrO}_{2}$ particles. 


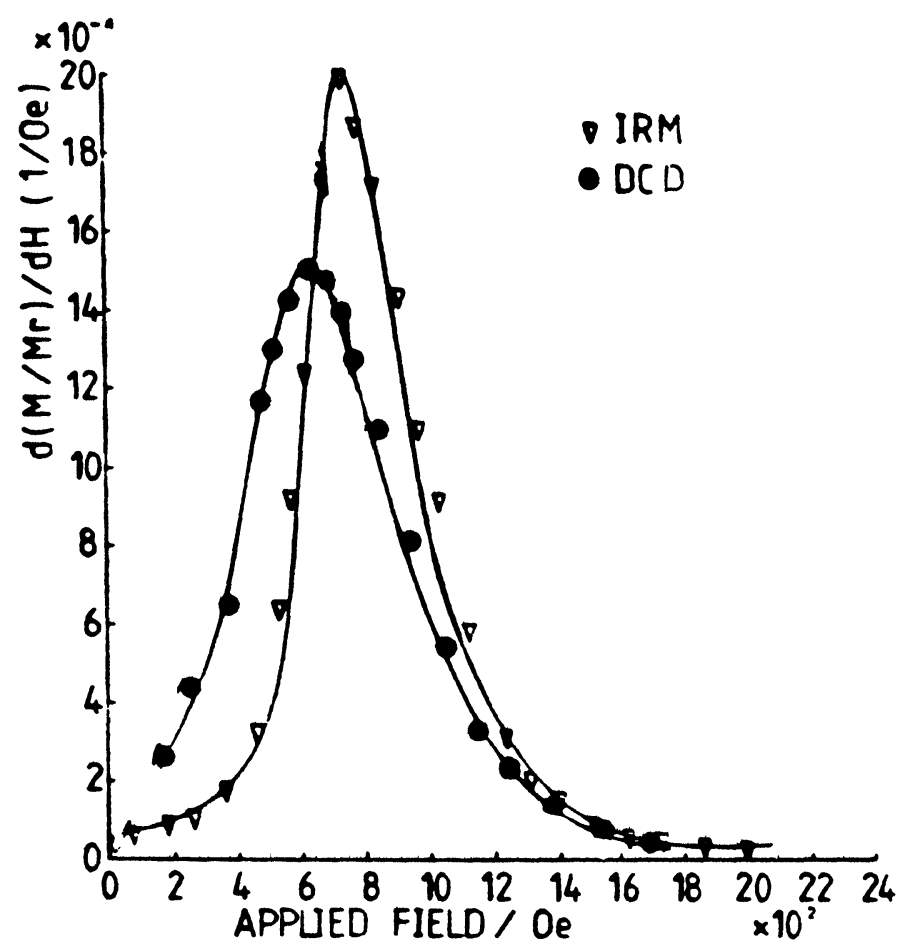

Figure 11 The SFD calculated by differentiation of the IRM curve given in Figure 10.

have studied NdFe alloys, finding in general non-linear behaviour. Spratt et $_{\text {al. }}{ }^{24}$ and Bissell et al. ${ }^{25}$ have carried out measurements on powders of $\mathrm{CrO}_{2}$ particles and FeSi soft steels which are also highly non-linear. In both cases the deviation from linearity is concave upwards, as illustrated in Figure 12 which shows data obtained for a FeSi sample (after Bissell et al. ${ }^{25}$ ).

This has the opposite sense to the deviation from linearity in the case of the hard materials. Typical data for NdFe (after Pinkerton ${ }^{22}$ ) are given in Figure 13.

The explanation for these results is most likely to lie in the many-body effects which are intrinsic to all magnetic materials and which are neglected in Eq. (21). Many-body effects are strongly dependent on the physical microstructure of a material and are thus characteristic of its texture. For example a recording medium whose particles have been aligned by the application of a magnetic field during the production process might be expected to exhibit different interaction effects than a system which is isotropic. In this section we consider interactions in relation to the texture as represented by the physical microstructure.

In this respect calculations on model systems are very useful in providing a basis for the understanding of experimental data. Here we give the preliminary results of a computational study using a model developed by Lyberatos et al. ${ }^{26}$ Here calculations are carried out on a cell of 1000 particles situated on a lattice. At each stage in the magnetisation process a particle is allowed to reverse its 


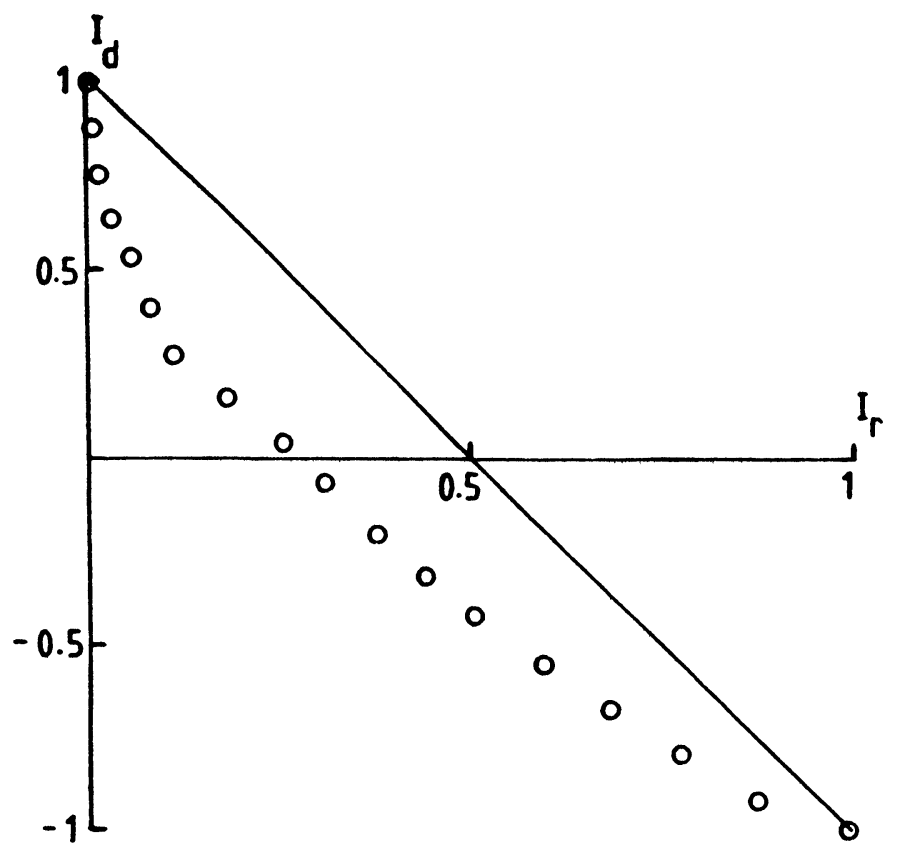

Figure 12 Henkel plot for a FeSi powder sample.

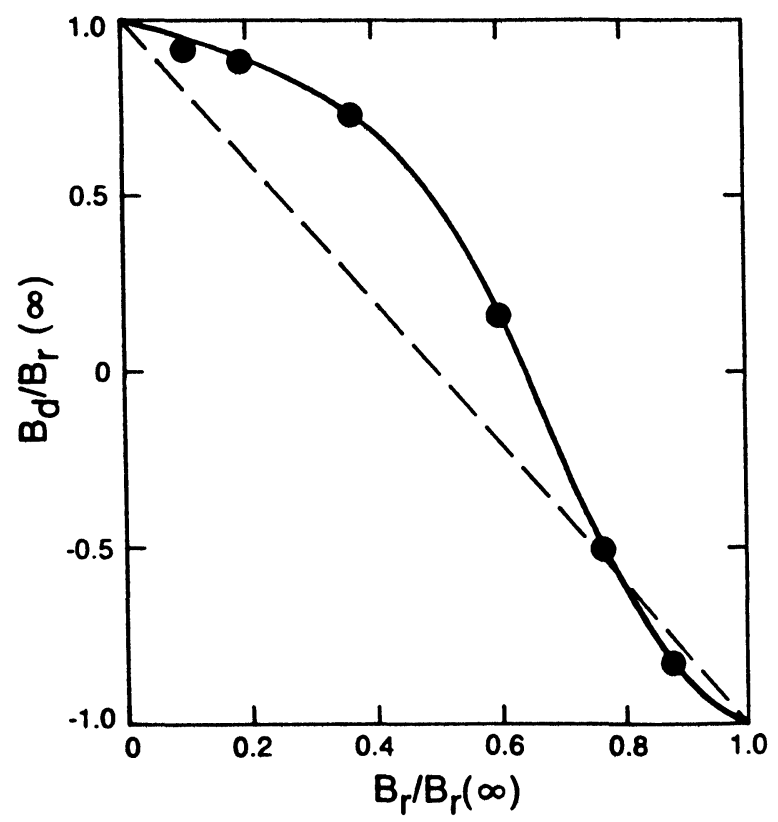

Figure 13 Henkel plot for NdFe. 
magnetisation with a probability given by

$$
p=1-\exp (-t / \tau)
$$

where $t$ is the time elapsed after some change in the external magnetic parameters and $\tau$ is a relaxation time given by the Arrhenius-Néel law ${ }^{27}$

$$
\tau^{-1}=f_{0} \exp \left(-\frac{K V}{k T}\left(1-H_{\mathrm{loc}} / H_{k}\right)\right)
$$

Here the frequency factor $f_{0} \sim 10^{9} \mathrm{~s}^{-1}, V$ is the particle volume, $K$ is the anisotropy constant and $H_{k}=2 K / I_{s}$ is the anisotropy field. Interaction effects are included via the term $H_{\text {loc }}$, the local field which can be written

$$
H_{\mathrm{loc}}=H_{a}+\sum_{i \neq j} H_{i j}+N_{d} I \text {, }
$$

where $H_{a}$ is the applied field. The second term represents the summation of the interaction fields over the nearest neighbours and the final term is a mean field taking into account the long-range interactions.

It is assumed that the particles have their easy axes perfectly oriented, so that (at the particle sizes studied here) there are essentially no reversible magnetisation changes. Hence the hysteresis loops are rather square, the rounding of the loop arising from the intrinsic SFD of the system which is due to the particle size distribution (assumed Gaussian) of the particles. $H_{\mathrm{loc}}$ has an important contribution from the nearest neighbour interaction field term which has a strong bearing on the magnetic behaviour of the system. An important factor here is the existence of very large spatial and temporal fluctuations in the local field which tend to drive the system towards thermal equilibrium. ${ }^{26}$ Another effect of the fluctuations is in increase the effective width of the SFD thereby reducing the squareness of the hysteresis loop.

The "Henkel plot" of $I_{d}$ vs $I_{r}$ provides useful information on the many-body effects. Figure 14 shows the calculated Henkel plot for a system of $0.02 \mu \mathrm{m}$ diameter cobalt particles with a Gaussian distribution of particle diameters having a standard deviation of 0.15 . The starting state for the IRM is taken as having moments oriented at random (consistent with the production of a demagnetised state). It can be seen that the plot is highly non-linear and is concave upwards consistent with experimental data on $\mathrm{CrO}_{2}$ particles. This form of plot is characteristic of systems in which the dipolar interaction has a net demagnetising effect. A very different form is observed for a lattice consisting essentially of isolated chains, as shown in Figure 15.

The tetragonal lattice has a microstructure consisting of sets of planes, which is very distinct from that of the chain lattice. The difference between these two types of spatial order, characteristic of the texture of the system, is strongly reflected in the form of the Henkel plot. This, consequently seems a promising device for studying for studying many-body effects in relation to the texture of a fine particle system. It is reasonable to conjecture that similar conclusions may apply to other materials exhibiting non-linear Henkel plots. Further work is in progress in order to examine this possibility.

It is interesting to note that the IRM curve and, consequently the Henkel plot is dependent on the initial configuration. For example, in the case of a cubic 


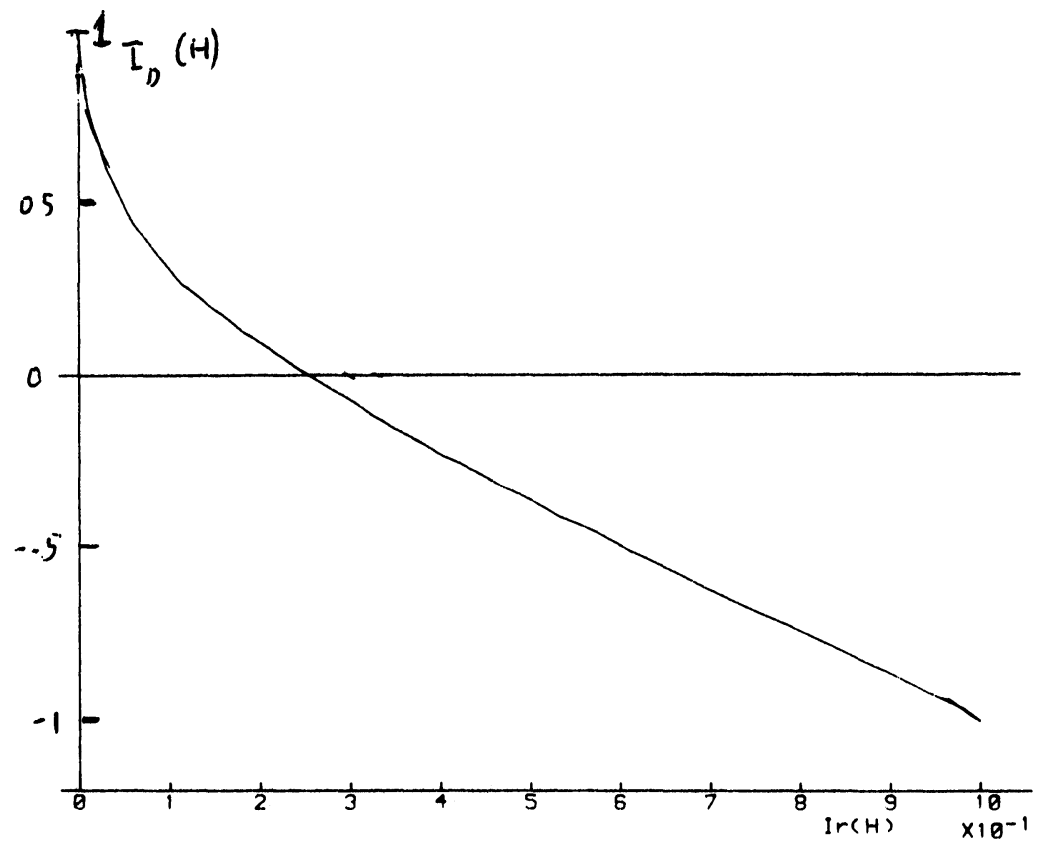

Figure 14 Henkel plot for a system of cobalt particles on a tetragonal lattice.

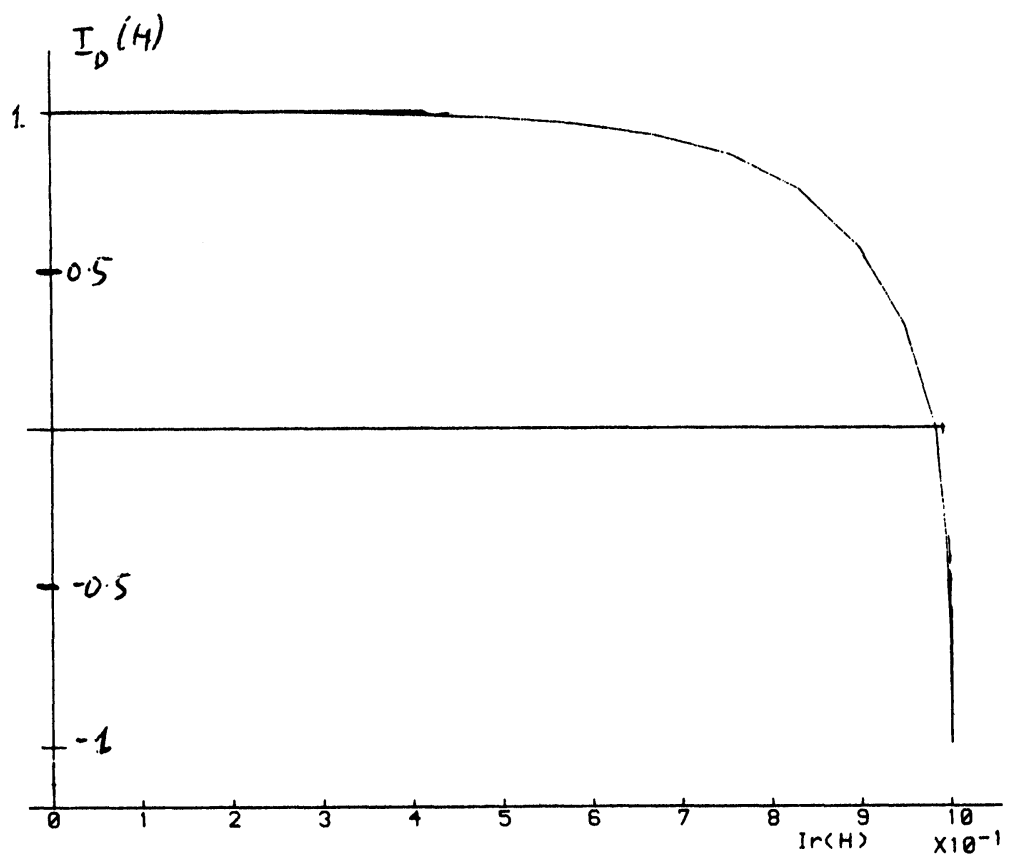

Figure 15 Henkel plot for a lattice of isolated chains in which interactions have a net magnetising effect. 
lattice, ac demagnetisation completely changed the sign of the deviation from linearity of the plot relative to the case of a completely random initial configuration. Thus the interpretation of Henkel plots should be carried out with some care. However the dependence of the IRM curves on the initial state is also of some interest in its own right in the investigation of interaction effects. This is presently being investigated theoretically and experimentally.

\section{CONCLUSION}

The effects of texture have an important effect on the behaviour of fine particle systems. Here we have considered the behaviour of two important classes of materials: Ferrofluids and particulate recording media. In both cases two types of texture can be identified. These are

1. Orientation texture. This is characteristic of the easy axis distribution of the particle anisotropy.

2. Spatial texture. This is characteristic of the physical microstructure of the material under investigation, which can be highly anisotropic and, in the case of a ferrofluid, strongly dependent on the magnitude of the applied magnetic field.

In ferrofluids both types of texture occur and are likely to contribute to the anisotropic properties of the materials. In highly stable systems it is likely that both mechanisms exist simultaneously. In cases such as the composite materials consisting of dispersions of polystyrene spheres in a ferrofluid, ${ }^{28}$ the spatial texture is predominant, making them excellent model systems for the study of field-induced anisotropy. ${ }^{29}$ Other systems of this type are very strongly interacting ferrofluids whose behaviour are strongly affected by the "macrochains" studied recently by Jones and Niedoba. ${ }^{30}$

The behaviour of solid dispersions of fine particles are also strongly affected by both types of texture. Not discussed here, but of considerable importance is the effect of the orientational texture on the magnetic behaviour of recording media. This is evident both in the coercivity of the medium and in the width of the SFD. ${ }^{31}$ Both of these parameters have an important bearing on the practical performance of recording media. Recent work ${ }^{32}$ has shown that interactions, and as a consequence the texture of the material, play an important role in determining the behaviour of fine particle systems. This shows that the physical microstructure of the material plays an important role in determining its practical behaviour. Much more work needs to be done in order to elucidate this role in order to fully optimise the properties of particulate recording media.

\section{ACKNOWLEDGEMENTS}

I am grateful to many colleagues for helpful discussions, particularly K. O'Grady, P. R. Bissell and the late Peter Wohlfarth. I am also grateful to Mr. M. Fearon for the computational work on remanence curves described here. 


\section{References}

1. Stoner, E. C. and Wohlfarth, E. P. (1948). Proc. Roy Soc. A240, 599.

2. Chantrell, R. W. (1986). J. Magn. Magn. Mater., 45, 100.

3. Krueger, D. A. (1983). J. Appl. Phys., 50, 8169.

4. Chantrell, R. W., Hoon, S. R. and Tanner, B. K. (1983). J. Magn. Magn. Mater., 38, 83.

5. Scholten, P. C. (1980). IEEE Trans. Mag., MAG-16, 221.

6. Llewellyn, J. P. (1983). J. Phys. D, 16, 95.

7. Mailfert, A. J. and Nahounou, B. (1980). IEEE Trans. Mag., MAG-16, 254.

8. Colteu, A. (1983). J. Magn. Magn. Mater., 39, 88.

9. Liebert, L. Martinet, A. and Strzelecki, L. (1972). J. Coll. and Interface Sci., 41, 391.

10. Binder, K. (1979). Monte-Carlo Methods in Statistical Physics, Springer-Verlag.

11. Chantrell, R. W., Bradbury, A. Popplewell, J. and Charles, S. W. (1982). J. Appl. Phys., 53, 2742.

12. Chantrell, R. W. (1985). J. de Phys. Coll. C6, 271.

13. Ayoub, N. Y., Bradbury, A., Chantrell, R. W. and Popplewell, J. (1987). J. Magn. Magn. Mater., 65, 185.

14. Martin, G. A. R., Bradbury, A. and Chantrell, R. W. (1986). IEEE Trans. Mag. MAG-22, 1137.

15. Bean, C. P. and Livingston, J. D. (1959). J. Appl. Phys., 30, 120 S.

16. Ayoub, N. Y., El-Hilo, M., Laham, N., Chantrell, R. W. and Popplewell, J. (1988). J. Phys. D 21, 1291.

17. O'Grady, K., Bradbury, A., Popplewell, J., Charles, S. W. and Chantrell, R. W. (1985) J. Magn. Magn. Mater., 49, 106.

18. Spratt, G. W. D., Bissell, P. R. and Chantrell, R. W. (1987). IEEE Trans. Mag., MAG-23, 186.

19. Wohlfarth, E. P. (1958). J. Appl. Phys., 29, 595.

20. Henkel, O. (1964). Phys. Stat. Sol. 7, 919.

21. Gaunt, P., Hadjipanayis, G. and Ng, C. (1986). J. Magn. Magn. Mater., 54-57, 841.

22. Pinkerton, F. E. (1986). IEEE Trans. Mag. MAG-22, 922.

23. Pinkerton, F. E. and Van Wingerden, D. J. (1986). J. Appl. Phys., 60, 3685.

24. Spratt, G. W. D., Bissell, P. R., Chantrell, R. W. and Wohlfarth, E. P. (1988). J. Magn. Magn. Mater., 75, 39.

25. Bissell, P. R., Chantrell, R. W., Spratt, G. W. D., Lutke-Stetzkamp, H. J., Methfessel, S. and Wohlfarth, E. P. J. de Phys (in press).

26. Lyberatos, A., Wohlfarth, E. P. and Chantrell, R. W. (1985). IEEE Trans. Mag. MAG-21, 1277.

27. Néel, L. (1949). Ann Geophys., 5, 99.

28. Skjeltorp, A. T. (1983). Phys. Rev. Lett., 51, 2036.

29. Davies, P., Popplewell, J., Martin, G. A. R., Bradbury, A. and Chantrell, R. W. (1986). J. Phys. $D, 19,469$.

30. Jones, G. A. and Niedoba, H. (1988). J. Magn. Magn. Mater., 73, 33.

31. Chantrell, R. W., O'Grady, K., Bradbury, A., Charles, S. W. and Hopkins, N. (1987). IEEE Trans. Mag., MAG-23, 204.

32. Fearon, M., Chantrell, R. W. and Wohlfarth, E. P. to be published. 